\title{
Protective Effect of Cytosolic Phospholipase A2 Inhibition against Inflammation and Degeneration by Promoting Regulatory $T$ Cells in Rats with Experimental Autoimmune Encephalomyelitis
}

\author{
Dan Yang, ${ }^{1}$ Hong-Fei Ji, ${ }^{2}$ Xue-Mei Zhang, ${ }^{1}$ Hui Yue, ${ }^{1}$ Lin Lin, ${ }^{1}$ Yu-Yan Ma, \\ Xiang-nan Huang, ${ }^{1}$ Jin Fu, ${ }^{1}$ and Wei-Zhi Wang ${ }^{1}$ \\ ${ }^{1}$ Department of Neurology, Second Affiliated Hospital, Harbin Medical University, 246 Xuefu Road, Nangang District, Harbin 150086, \\ China \\ ${ }^{2}$ Department of Molecular Biology and Genetic Engineering, Cancer Institute of Heilongjiang Province, \\ Harbin Medical University, Harbin 150081, China
}

Correspondence should be addressed to Jin Fu; fujin6777@163.com and Wei-Zhi Wang; wangwzhhmu@yeah.net

Received 17 October 2013; Accepted 28 January 2014; Published 23 March 2014

Academic Editor: Dianne Cooper

Copyright (C) 2014 Dan Yang et al. This is an open access article distributed under the Creative Commons Attribution License, which permits unrestricted use, distribution, and reproduction in any medium, provided the original work is properly cited.

Cytosolic phospholipase A2 $\left(\mathrm{CPLA}_{2}\right)$ is the rate-limiting enzyme that initiates the production of various inflammatory mediators. Previous studies have shown that inhibiting $\mathrm{CPLA}_{2}$ exerts a neuroprotective effect on experimental autoimmune encephalomyelitis (EAE) by ameliorating the severity of the disease and influencing Th1 and Th17 responses. However, it remains unclear whether treatment with a cPLA 2 inhibitor will influence the regulatory $\mathrm{T}$ cells (Tregs) that play a critical role in maintaining immune homeostasis and preventing autoimmune diseases. In this study, the $\mathrm{CPLA}_{2}$ inhibitor AX059 reduced the onset and progression of EAE in Lewis rats. In addition, this effect was accompanied by activation of Tregs and alterations in the expression of their various cytokines. The study therefore demonstrated that Tregs are involved in the immunomodulatory effect mediated by $\mathrm{cPLA}_{2}$ inhibition. These findings may have clinical application in the treatment of multiple sclerosis.

\section{Introduction}

Multiple sclerosis (MS) is an inflammatory, demyelinating disorder of the central nervous system (CNS) affecting over 2.5 million young adults worldwide. Much of our understanding of the pathogenesis of MS is based on studies using the animal model, experimental autoimmune encephalomyelitis (EAE) [1]. Many variations of EAE are now available; for example, Lewis rats can develop monophasic or chronic EAE after injection of myelin basic protein (MBP), and this is a stable animal model to explore the mechanisms underlying CNS autoimmune diseases $[2,3]$.

The mechanisms underlying the pathogenesis of MS or EAE were previously believed to be mediated mainly by Th1 cells and Th1-related cytokines, which initiate a pathogenic response directed against the components of CNS myelin and lead to inflammation, demyelination, axonal damage, and, ultimately, functional deficits [4]. Nevertheless, many recent reports have suggested that abnormal regulatory $\mathrm{T}$ cells (Tregs) are involved in the pathogenesis of autoimmune demyelination in EAE and MS $[5,6]$. Tregs are a subpopulation of $\mathrm{CD} 4+\mathrm{T}$ cells which are central to the acquisition and maintenance of immunological self-tolerance, as well as tolerance of tissue grafts and prevention of autoimmune diseases [7]. CD25 has been identified as a phenotypic marker for Tregs, and the forkhead/winged helix transcription factor forkhead box P3 (Foxp3) is its specific transcription factor. In patients with MS, a functional defect of Tregs has been 
found [8]. In EAE, Tregs administered to mice can also significantly reduce EAE severity $[9,10]$ and have been shown to accumulate within the CNS during recovery [11].

In addition to autoreactive $\mathrm{T}$ cells and inflammatory cytokines, various other mediators of inflammation that recruit and modulate immune cells play major roles in the pathogenesis of these disorders. Phospholipase A2 $\left(\mathrm{PLA}_{2}\right)$ is a heterogeneous group of enzymes that specifically hydrolyze fatty acids at the sn- 2 position of cell membrane phospholipids, which gives rise to eicosanoids that contribute to various aspects of inflammation through cyclooxygenase (COX) and lipoxygenase pathways $[12,13]$. Among the various $\mathrm{PLA}_{2}$ enzymes, cytosolic PLA2 $\left(\mathrm{cPLA}_{2}\right)$ is the predominant isoform with enzymatic activity, and $\mathrm{CPLA}_{2}$ plays important roles in the arachidonic acid cascade [14]. Previous studies have suggested that $\mathrm{CPLA}_{2}$ participates in EAE development and that inhibition of $\mathrm{CPLA}_{2}$ may be valuable for the prevention EAE, which suggests that $\mathrm{CPLA}_{2}$ inhibitors may be potentially useful immunomodulators in the treatment of MS $[15,16]$. Furthermore, one study in a mouse EAE model has shown that the involvement of $\mathrm{CPLA}_{2}$ in the pathogenesis of EAE is associated with Th17-type responses [17]. However, there are no reports describing the correlation of $\mathrm{CPLA}_{2}$-mediated neuroinflammation and neurodegeneration with Tregs.

In the present study, we investigated for the first time the effect of $\mathrm{CPLA}_{2}$ in rats with EAE by using a selective $\mathrm{cPLA}_{2}$ inhibitor. Our findings indicate that blocking $\mathrm{CPLA}_{2}$ can reduce the onset and progression of the condition. In addition, we found that Tregs and their regulatory cytokines are altered when $\mathrm{CPLA}_{2}$ is blocked in rats with EAE.

\section{Materials and Methods}

2.1. Animals. Female Lewis rats ( 8 weeks old) weighing approximately $160-170 \mathrm{~g}$ were obtained from Beijing Vital River Laboratory Animal Co. Ltd (Beijing, China). All rats were bred in specific pathogen-free and climate-controlled conditions. All in vivo experiments in these animals were performed in accordance with the Committee on Ethics of Animal Experiments, Harbin Medical University.

2.2. EAE Induction. EAE was induced as previously described [18]. Briefly, rats were immunized with $25 \mu \mathrm{g}$ of myelin basic protein MBP (Sigma) emulsified with complete Freund's adjuvant (CFA) containing $5 \mathrm{mg} / \mathrm{mL}$ of Mycobacterium butyricum (Sigma). This solution $(100 \mu \mathrm{L})$ was injected subcutaneously into the footpad of the animals' hind limbs. Rats injected with the vehicle solution were used as control. The rats were evaluated daily for weight loss and scored for neurological impairment as follows: $0=$ no signs; $1=$ loss of tonicity of the distal portion of the tail; $2=$ total loss of tail tonicity; 3 = hind limb weakness (partial paralysis); $4=$ complete hind limb paralysis and urinary incontinence; $5=$ dead.

2.3. Drug Treatment. Rats were randomly assigned to either the treatment or control group and were treated with either the 2-oxoamide $\mathrm{CPLA}_{2}$ inhibitor AX059 (intraperitoneal injections of $200 \mu \mathrm{L}$ of a $4 \mathrm{mmol} / \mathrm{L}$ solution) or vehicle (PBS containing $1 \%$ dimethyl sulfoxide) administered once daily for 2 weeks, starting on the day of immunization for EAE induction. The synthesis of AX059 was in accordance with the method of Stephens et al. [19] and Barbayianni et al. [20]. Monitoring was performed on a blinded basis so that the person doing the scoring was unaware of the experimental group that the animals had been assigned to.

2.4. Histological Analysis. Rats were deeply anesthetized by inhalation of $5 \%$ carbon dioxide on day 14 . After euthanasia and blood withdrawal, brain and spinal cord samples were dissected and fixed in $4 \%$ paraformaldehyde before embedding in paraffin. Selected panels of serial sections $(4-5 \mu \mathrm{m})$ were processed for routine hematoxylin and eosin (H\&E) staining, Luxol fast blue staining, and immunohistochemical labeling with anti-Foxp3 and analyzed with a Nikon microscope. The number of inflammatory foci containing at least 20 cells was counted in each H\&E-stained section in a blinded fashion by the same pathologist.

2.5. Flow Cytometry Analysis. To analyze the prevalence of Tregs, cellular surface markers were evaluated via flow cytometry using the following antibodies: fluorescein isothiocyanate (FITC) conjugated CD4 and phycoerythrin (PE) conjugated CD25 (all purchased from BD Biosciences Pharmingen, San Diego, CA). Lymph cells were washed with cold phosphate-buffered saline (PBS) and resuspended in further $100 \mathrm{~mL}$ of PBS. Cells were stained for $20 \mathrm{~min}$ at $4^{\circ} \mathrm{C}$ with FITC-CD4 and PE-CD25 antibodies. Staining was visualized on an FACS Calibur flow cytometer (BD, Franklin Lakes, NJ, USA) with Cell Quest (version 3.2.1f1, BD).

2.6. Assessment of Cytokine Production. To assess cytokine production, spleens were isolated from rats in each treatment group. Mononuclear cell suspensions were prepared and $2 \times$ $10^{6}$ cells were cultured in complete RPMI medium and initiation with MBP. The cells were then treated with either AX059 $(30 \mu \mathrm{mol} / \mathrm{L})$ or vehicle for 60 hours. Supernatant obtained from in vitro cultures was analyzed for interleukin6 (IL-6), interleukin-10 (IL-10), and transforming growth factor- $\beta$ (TGF- $\beta$ ) according to the manufacturer's protocol (BD Bioscience). For the FACS experiment, lymphocytes were used.

2.7. Statistical Analysis. Data were expressed as means \pm standard deviation, and statistical differences in the severity of neurological impairment, inflammatory foci, Foxp3 expression, flow cytometry data, and cytokine production between the various groups were determined by analysis of variance (ANOVA) and the Mann-Whitney rank-sum test. Statistical analyses were performed using SPSS for Windows (version 11.0, SPSS Inc., Chicago, USA). A $P$ value less than 0.05 was considered statistically significant. 


\section{Results}

3.1. Effect of $c P L A_{2}$ Inhibition on the Severity of EAE in Lewis Rats. To investigate the effect of the $\mathrm{CPLA}_{2}$ inhibitor AX059 on the development of EAE, Lewis rats were treated separately with AX059 and vehicle. The rats treated with AX059 were resistant to EAE induction; 4 of 12 rats in this group developed EAE as compared with all 12 rats treated with vehicle. In addition, the average maximum neurological impairment score was significantly reduced when compared with the vehicle treatment group, the peak neurological impairment occurred 2 days later, and the remaining neurological impairment time was shorter, indicating that inhibition of $\mathrm{CPLA}_{2}$ delayed the onset of EAE and hastened its recovery (Figure 1).

To further assess inflammation in the central nervous system, the pathological changes revealed by H\&E staining of the animals' brains and spinal cords were consistent with the neurological impairment scores. Inflammatory cells infiltrated around blood vessels in the leptomeninges and white matter and generated several focal lesions of inflammation that were related to the severity of EAE. Pathological changes in the spinal cord were more marked than those in the brain.

Demyelination was also evaluated in Luxol fast blue sections of the spinal cord. Although obvious signs of demyelination within white matter were observed in vehicletreated rats, only minimal changes were observed in AX059treated rats (Figure 2). Microscopic examination found that there were $17.2 \pm 2.7$ and $14.7 \pm 2.1$ focal lesions in AX059treated rats' brains and spinal cords, respectively, whereas $7.1 \pm 1.9$ and $4.3 \pm 1.5$ in rats treated separately with vehicle. The number of focal lesions in AX059-treated rats was significantly less than in vehicle-treated rats (Figure 3 ).

3.2. Effect of $c P L A_{2}$ Inhibition on Tregs. To analyze the effect of $\mathrm{cPLA}_{2}$ inhibitor treatment on Tregs, monocytes were isolated from the spleens of rats in the 2 treatment groups at 14 days, and expression levels of CD4 and CD25 on the cells' surfaces were measured by flow cytometry. Compared with rats in the vehicle-treated group, AX059-treated rats showed markedly increased Tregs, and the difference in the percentages of Tregs $(5.9 \pm 1.0 \%$ versus $1.3 \pm 0.7 \%)$ between the 2 groups (Figure 4$)$ reached statistical significance $(P<0.05)$.

To further investigate functional changes of Tregs in the lesions, including both the brain and spinal cord, we used an immunocytochemical method to assess the expression of Foxp3, which is critical for the regulatory function of Tregs and is also a unique marker for their identification. As shown in Figure 2, Tregs were located in both the white and grey matter of the brain and spinal cord and were present in larger numbers in rats treated with AX059 than in vehicle-treated rats (Figures 2(d) and 2(h)). There was little infiltration of $\mathrm{T}$ cells and minimal myelin and axonal damage in the location of Tregs homing.

3.3. Effect of $c P L A_{2}$ Inhibition on the Cytokine Expression of Tregs. To explore the mechanisms underlying the effect of cPLA $_{2}$ inhibition on Tregs, we analyzed 3 cytokines, including TGF- $\beta$, IL-10, and IL-6, which are closely associated with

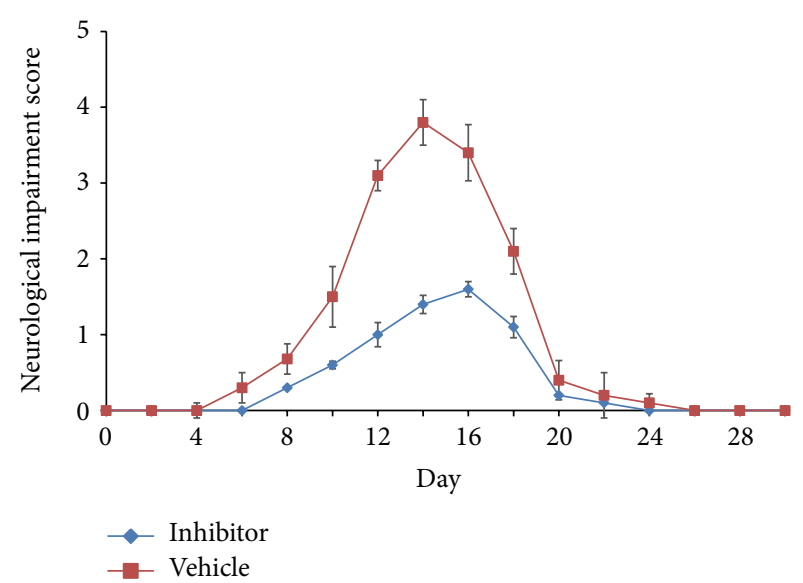

FIGURE 1: Neurological impairment scores in Lewis rats with experimental autoimmune encephalomyelitis (EAE). The rats were immunized with $25 \mu \mathrm{g}$ of myelin basic protein (MBP) and $5 \mathrm{mg} / \mathrm{mL}$ of Mycobacterium butyricum and then treated with either the $\mathrm{CPLA}_{2}$ inhibitor AX059 or vehicle once daily, starting on the day of immunization for EAE induction. EAE development in the rats was assessed on a scale of $0-5$.

the functions of Tregs. The immunosuppressive cytokines TGF- $\beta$ and IL-10 were increased in AX059-treated rats with EAE as compared with vehicle-treated rats. Conversely, a striking reduction was observed in the typical inflammatory cytokine IL-6 (Figure 5). Together, these findings indicate that AX059 induced the activation and proliferation of Tregs to interfere with the pathological immune response. The data suggest that changes in these 3 cytokines play a pivotal role in the induction of Tregs by AX059 and subsequently in the diminished infiltration of inflammatory cells and the neuroprotection afforded by AX059.

\section{Discussion}

As the key esterase involved in the synthesis of many inflammatory mediators, $\mathrm{cPLA}_{2}$ has been known to play extensive roles in autoimmune and oxidative stress in neurological disorders [21]. Recent studies have demonstrated that $\mathrm{CPLA}_{2}$ serves as a central mediator of EAE and MS development, the potential mechanism of which includes not only initiating the secretion of inflammatory effectors and chemokines, but also promoting immune cell infiltration, demyelination, and

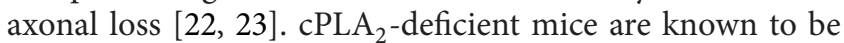
resistant to EAE, and inhibition of $\mathrm{CPLA}_{2}$ exerts an antiinflammatory effect in mice with EAE by delaying or reducing the onset and progression of the disease [24]. Therefore, $\mathrm{CPLA}_{2}$ is believed to be important in the pathogenesis of MS, and blockade of the enzyme might have therapeutic benefits in reducing its progression.

Rat strains differ from mice in their susceptibility to the disease, which gives us another insight into human MS. In the present study, we showed that the $\mathrm{CPLA}_{2}$ inhibitor AX059 played a protective role in the Lewis rat EAE model, decreasing the incidence, delaying the peak of neurological impairment, and reducing the severity of the condition. 


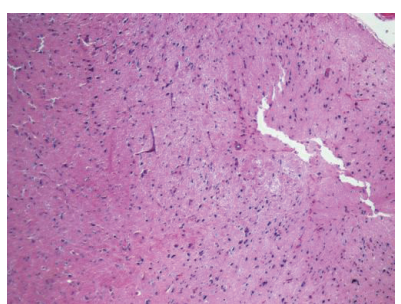

(a)

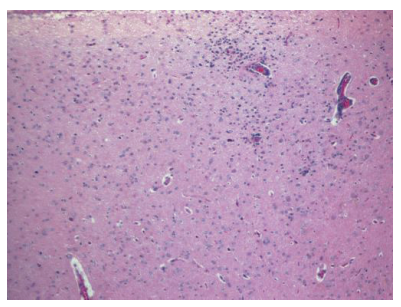

(e)

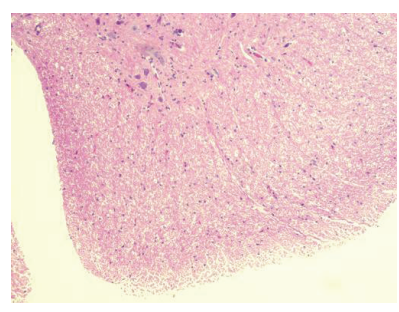

(b)

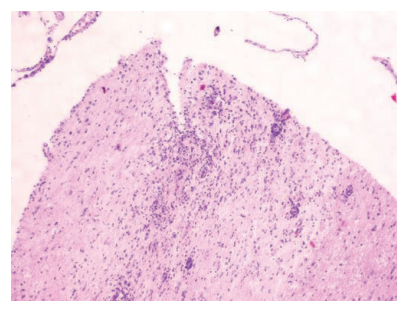

(f)

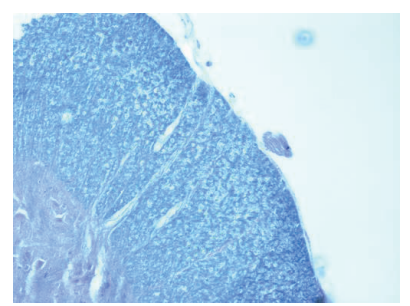

(c)

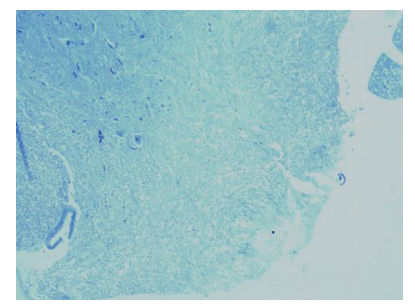

(g)

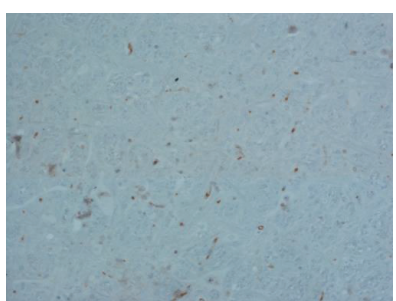

(d)

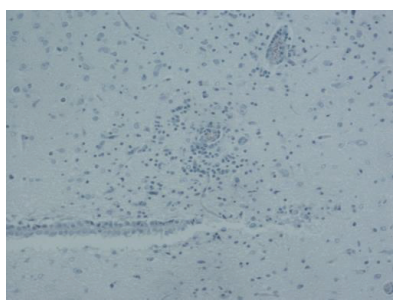

(h)

FIGURE 2: Treatment with the CPLA 2 inhibitor AX059 reduced brain and spinal cord damage in EAE induced in Lewis rats. The figures shows hematoxylin and eosin (H\&E)-stained sections from AX059-treated ((a) brain; (b) spinal cord) and vehicle-treated ((e) brain; (f) spinal cord) rats; spinal cord sections from AX059-treated (c) or vehicle-treated (g) rats stained with Luxol fast blue; and Foxp3 expression in spinal cord sections from AX059-treated (d) or vehicle-treated (h) rats by immunohistochemical staining.

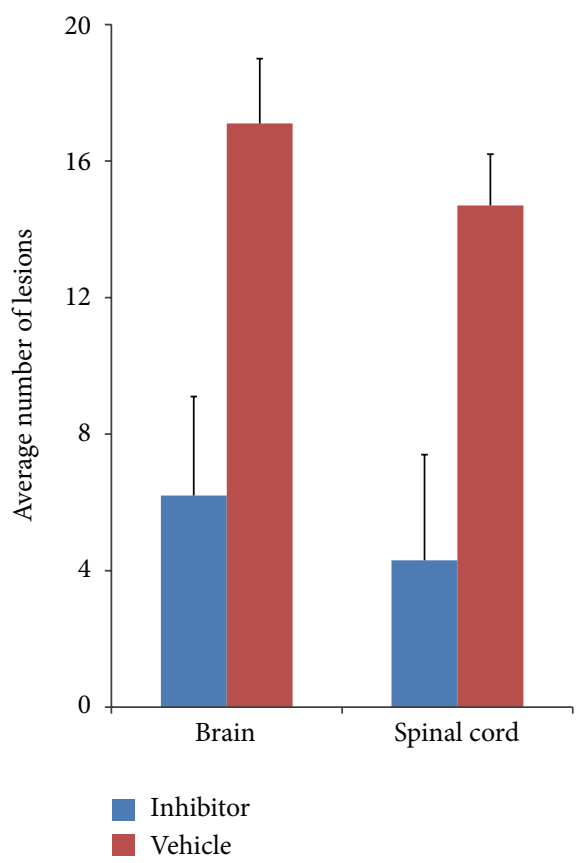

FIGURE 3: Morphometric analysis of infiltrated lesions in the brains and spinal cords of AX059-treated and vehicle-treated rats.

Furthermore, treatment with AX059 induced activation of Tregs and enhanced their numbers. Our findings therefore suggest that Tregs are involved in the anti-inflammatory effect of $\mathrm{CPLA}_{2}$ inhibition in the rat EAE model.

Previous studies have shown that $\mathrm{CPLA}_{2}$ has an important role in the regulation of $\mathrm{T}$ cell differentiation during $\mathrm{EAE}$ development and that blocking $\mathrm{CPLA}_{2}$ with an inhibitor or by gene knockout prevents inflammatory cell production $[17,25,26]$. Marusic et al. reported that $\mathrm{CPLA}_{2 \alpha}^{-/-}$mice develop impaired Th1-type responses [24], while another study revealed that blockade of $\mathrm{CPLA}_{2}$ led to diminished Th1type and Th17-type cytokines, as well as striking reductions in interferon- (IFN-) $\gamma$, tumor necrosis factor (TNF) and IL-17 concentrations [17]. Taketomi et al. also showed that $\mathrm{CPLA}_{2}$ drives mast cell maturation in anaphylaxis [27]. Based on these findings and the fact that $\mathrm{CPLA}_{2}$ inhibition was closely associated with multiple aspects of EAE pathogenesis and various types of $\mathrm{T}$ cell responses in the present study, it is worth determining whether treatment with a $\mathrm{CPLA}_{2}$ inhibitor will alter the abundance and function of Tregs, which are the key regulators of lymphocyte proliferation and differentiation.

In our study, an increase in the percentage of Tregs was observed in Lewis rats with EAE after treatment with AX059 in comparison with rats treated with vehicle by using flow cytometry. To further evaluate whether the Tregs exhibited an immunosuppressive effect, we assessed the expression of Foxp3, a specific transcription factor that exerts immunosuppressive functions. Brain and spinal cord lesions were infiltrated by significantly higher numbers of Tregs in AX059-treated rats compared with vehicle-treated rats, and activated Tregs were induced by the inhibition of $\mathrm{CPLA}_{2}$. To our knowledge, this is the first study to demonstrate an association between Tregs and $\mathrm{CPLA}_{2}$ inhibition. In agreement with our findings, treatments that ameliorate the course of EAE have been found to enhance the development of Tregs during recovery in mouse EAE models [28-30]. Furthermore, some immunomodulatory therapies approved for MS such as glucocorticoids, interferon- $\beta$, and glatiramer 


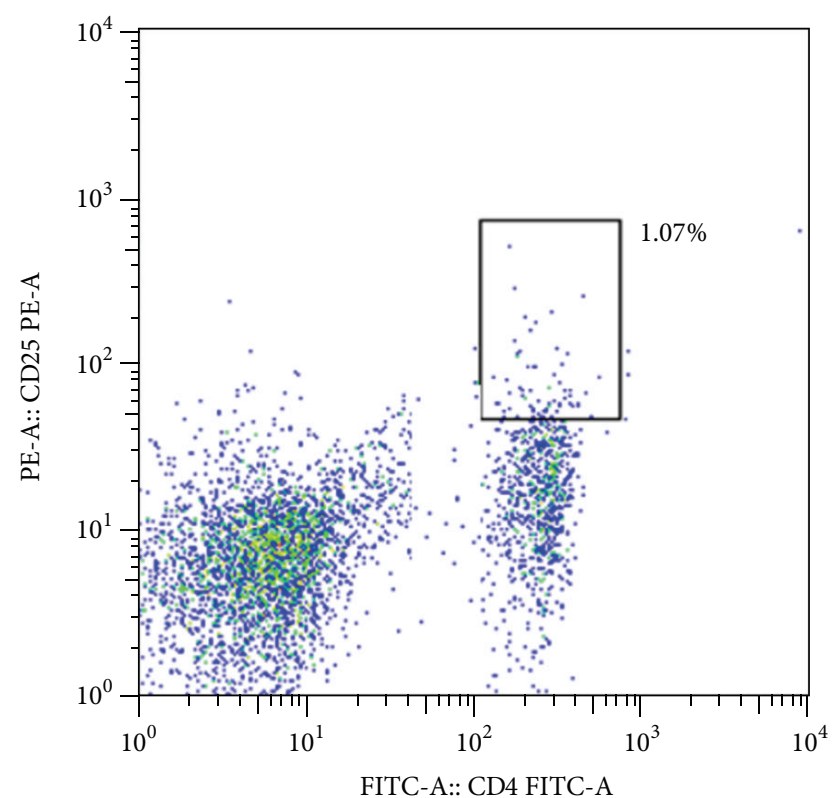

(a)

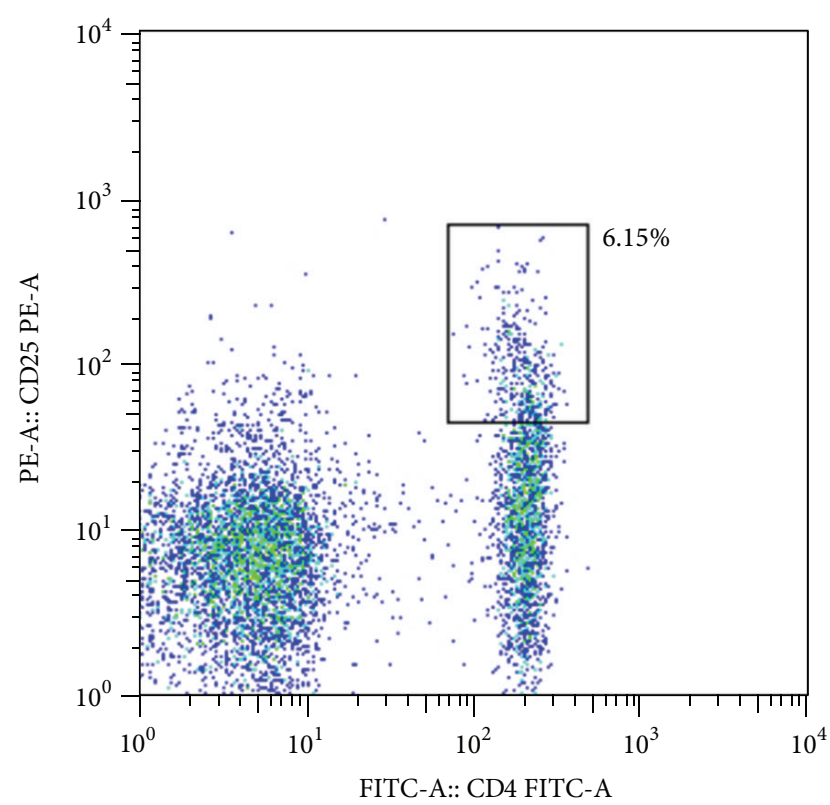

(b)

FIGURE 4: Rats treated with AX059 (b) displayed an increase in the percentage of Tregs during EAE induction compared with those treated with vehicle (a).

acetate have been found to have a significant effect on Tregs $[16,31-33]$. Thus, our findings may have clinical application in the treatment of MS.

The development and maintenance of the anti-inflammatory effects of Tregs are regulated by several cytokines, including TGF- $\beta$, IL-6, and IL-10 [34]. The development of Tregs is correlated with enhanced TGF- $\beta$ expression and reduced levels of IL-6. In addition to inducing differentiation

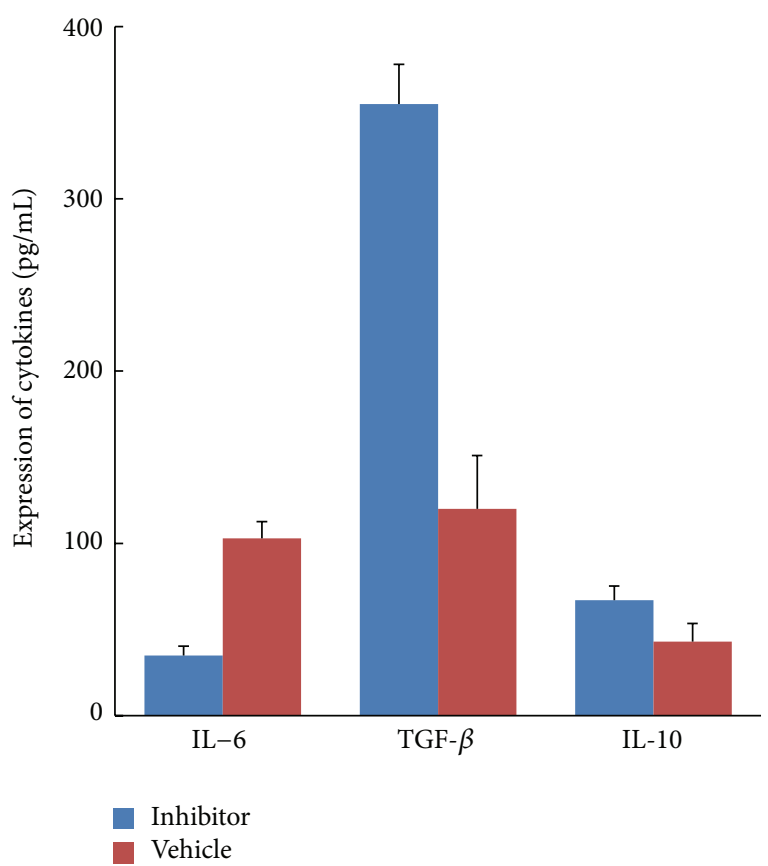

FIGURE 5: The $\mathrm{CPLA}_{2}$ inhibitor AX059 increased the expression of TGF- $\beta$ and IL-10 and downregulated the expression of IL- 6 in the rats' spleen cells.

and development of Tregs, TGF- $\beta$ also can prolong the halflife of Foxp3 RNA species and phosphorylate chromatinbound Foxp3 [35], and IL-6 plays a crucial role in regulating the balance between Treg cells and Th17 cells, which are involved in the pathogenesis of EAE and MS [36, 37]. At the same time, IL-6 can destroy the immunosuppressive function of Treg cells under inflammatory conditions $[38,39]$. Murai et al. reported that IL-10 was required to maintain a Treg-suppressive function and to maintain expression of the Foxp3 transcription factor [40]. Most recently, Klose reported that IL-10 production of transduced neural stem/progenitor cells (NSPC ${ }^{\mathrm{IL}-10}$ ) ameliorates the clinical disease course of $\mathrm{EAE}$, and the therapeutic mechanism was that NSPC ${ }^{\mathrm{IL}-10}$ suppressed IL- 2 and IFN- $\gamma$ production and did not induce a higher percentage of Tregs since IL-10 was produced in NSPC instead of lymphocyte [41]. On the other hand, previous studies have shown that cPLA regulates the production of cytokines, and blockade of cPLA ${ }_{2}$ causes suppression of Th1type and Th17-type cytokines $[15,17]$. Thus, it is conceivable that $\mathrm{CPLA}_{2}$ inhibition might play a central role by regulating the cytokine expression of Tregs. To test this hypothesis, we analyzed the expression of TGF- $\beta$, IL- 6 , and IL- 10 . When the cells were exposed to AX059, the positive modulatory effect of TGF- $\beta$ and IL-10 on Tregs differentiation increased. In contrast, the negative modulatory effect of IL-6 was significantly reduced. Previous studies have revealed that, whereas $\mathrm{CPLA}_{2}$ inhibition impairs the induction of Th1-type and Thl7-type cytokines, no report mentioned that it also makes contribution to increasing the production of Tregsrelated cytokines. 
In conclusion, our study in a rat model has demonstrated that $\mathrm{CPLA}_{2}$ may be involved in onset and progression of EAE and that $\mathrm{CPLA}_{2}$ inhibitor treatment may offer beneficial effects in reducing disease severity by regulating Tregs and mediators of their effects such as various cytokines and other T cells. Therefore, the development of selective $\mathrm{CPLA}_{2}$ inhibitors may provide hope for the treatment of human CNS autoimmune disease.

\section{Conflict of Interests}

The authors have no conflict of interests to disclose.

\section{Authors' Contribution}

Jin Fu, Wei-Zhi Wang, and Dan Yang designed this study. Dan Yang, Hong-Fei Ji, Yu-Yan Ma, and Xiang-nan Huang conducted the histological experiments, flow cytometry, and cytokine analysis. Xue-Mei Zhang, Lin Lin, and Hui Yue did the animal experiments. Dan Yang performed data analysis and drafted the paper. Wei-Zhi Wang and Jin Fu conceived the study and edited the paper. All authors read and approved the final paper. All authors critically reviewed the paper and approved the final version for submission.

\section{Acknowledgments}

This work was supported by the Natural Science Foundation of China (81171120/H0907, 81070964/H0907) and the Natural Science Foundation of Heilongjiang Province (D201108).

\section{References}

[1] A. Nylander and D. A. Hafler, "Multiple sclerosis," Journal of Clinical Investigation, vol. 122, no. 4, pp. 1180-1188, 2012.

[2] G. Krishnamoorthy and H. Wekerle, "EAE: an immunologist's magic eye," European Journal of Immunology, vol. 39, no. 8, pp. 2031-2035, 2009.

[3] K. O’Brien, B. Gran, and A. Rostami, “T-cell based immunotherapy in experimental autoimmune encephalomyelitis and multiple sclerosis," Immunotherapy, vol. 2, no. 1, pp. 99-115, 2010.

[4] E. M. Frohman, M. K. Racke, and C. S. Raine, "Medical progress: multiple sclerosis-the plaque and its pathogenesis," The New England Journal of Medicine, vol. 354, no. 9, pp. 942-955, 2006.

[5] D. E. Lowther and D. A. Hafler, "Regulatory T cells in the central nervous system," Immunological Reviews, vol. 248, no. 1, pp. 156169, 2012.

[6] M. Buc, "Role of regulatory T cells in pathogenesis and biological therapy of multiple sclerosis," Mediators of Inflammation, vol. 2013, Article ID 963748, 11 pages, 2013.

[7] K. Wing and S. Sakaguchi, "Regulatory T cells exert checks and balances on self tolerance and autoimmunity," Nature Immunology, vol. 11, no. 1, pp. 7-13, 2010.

[8] K. Venken, N. Hellings, R. Liblau, and P. Stinissen, "Disturbed regulatory $\mathrm{T}$ cell homeostasis in multiple sclerosis," Trends in Molecular Medicine, vol. 16, no. 2, pp. 58-68, 2010.

[9] J. Reddy, Z. Illes, X. Zhang et al., "Myelin proteolipid proteinspecific $\mathrm{CD} 4{ }^{+} \mathrm{CD} 25^{+}$regulatory cells mediate genetic resistance to experimental autoimmune encephalomyelitis," Proceedings of the National Academy of Sciences of the United States of America, vol. 101, no. 43, pp. 15434-15439, 2004.

[10] T. Korn, E. Bettelli, W. Gao et al., "IL-21 initiates an alternative pathway to induce proinflammatory T H17 cells," Nature, vol. 448, no. 7152, pp. 484-487, 2007.

[11] Y. Liu, I. Teige, B. Birnir, and S. Issazadeh-Navikas, "Neuronmediated generation of regulatory $\mathrm{T}$ cells from encephalitogenic T cells suppresses EAE," Nature Medicine, vol. 12, no. 5, pp. 518-525, 2006.

[12] J. E. Burke and E. A. Dennis, "Phospholipase A2 structure/function, mechanism, and signaling," Journal of Lipid Research, vol. 50, supplement, pp. S237-S242, 2009.

[13] V. Magrioti and G. Kokotos, "Phospholipase A2 inhibitors for the treatment of inflammatory diseases: a patent review (2010present)," Expert Opinion on Therapeutic Patents, vol. 23, no. 3, pp. 333-344, 2013.

[14] E. A. Dennis, J. Cao, Y.-H. Hsu, V. Magrioti, and G. Kokotos, "Phospholipase A2 enzymes: physical structure, biological function, disease implication, chemical inhibition, and therapeutic intervention," Chemical Reviews, vol. 111, no. 10, pp. 61306185, 2011.

[15] A. Kalyvas and S. David, "Cytosolic phospholipase A2 plays a key role in the pathogenesis of multiple sclerosis-like disease," Neuron, vol. 41, no. 3, pp. 323-335, 2004.

[16] S. G. Payne, C. A. Oskeritzian, R. Griffiths et al., "The immunosuppressant drug FTY720 inhibits cytosolic phospholipase A2 independently of sphingosine-1-phosphate receptors," Blood, vol. 109, no. 3, pp. 1077-1085, 2007.

[17] S. Marusic, P. Thakker, J. W. Pelker et al., "Blockade of cytosolic phospholipase A2 $\alpha$ prevents experimental autoimmune encephalomyelitis and diminishes development of Thl and Th17 responses," Journal of Neuroimmunology, vol. 204, no. 1-2, pp. 29-37, 2008.

[18] M. Mannie, R. H. Swanborg, and J. A. Stepaniak, "Experimental autoimmune encephalomyelitis in the rat," Current Protocols in Immunology, vol. 85, no. 15, pp. 589-612, 2009.

[19] D. Stephens, E. Barbayianni, V. Constantinou-Kokotou et al., "Differential inhibition of group IVA and group VIA phospholipases A2 by 2-oxoamides," Journal of Medicinal Chemistry, vol. 49, no. 9, pp. 2821-2828, 2006.

[20] E. Barbayianni, D. Stephens, A. Grkovich et al., "2-Oxoamide inhibitors of phospholipase A2 activity and cellular arachidonate release based on dipeptides and pseudodipeptides," Bioorganic and Medicinal Chemistry, vol. 17, no. 13, pp. 48334843, 2009.

[21] A. A. Farooqui, W.-Y. Ong, and L. A. Horrocks, "Inhibitors of brain phospholipase A2 activity: their neuropharmacological effects and therapeutic importance for the treatment of neurologic disorders," Pharmacological Reviews, vol. 58, no. 3, pp. 591-620, 2006.

[22] A. Kalyvas, C. Baskakis, V. Magrioti et al., "Differing roles for members of the phospholipase A2 superfamily in experimental autoimmune encephalomyelitis," Brain, vol. 132, no. 5, pp. 12211235, 2009.

[23] D. K. Nomura, B. E. Morrison, J. L. Blankman et al., "Endocannabinoid hydrolysis generates brain prostaglandins that promote neuroinflammation," Science, vol. 334 , no. 6057 , pp. 809 813, 2011. 
[24] S. Marusic, M. W. Leach, J. W. Pelker et al., "Cytosolic phospholipase A2 $\alpha$-deficient mice are resistant to experimental autoimmune encephalomyelitis," Journal of Experimental Medicine, vol. 202, no. 6, pp. 841-851, 2005.

[25] J. R. Sundaram, E. S. Chan, C. P. Poore et al., "Cdk5/p25-induced cytosolic PLA2-mediated lysophosphatidylcholine production regulates neuroinflammation and triggers neurodegeneration," Journal of Neuroscience, vol. 32, no. 3, pp. 1020-1034, 2012.

[26] P. Thakker, S. Marusic, N. L. Stedman et al., "Cytosolic phospholipase A2 $\alpha$ blockade abrogates disease during the tissue-damage effector phase of experimental autoimmune encephalomyelitis by its action on APCs," Journal of Immunology, vol. 187, no. 4, pp. 1986-1997, 2011.

[27] Y. Taketomi, N. Ueno, T. Kojima et al., "Mast cell maturation is driven via a group III phospholipase A2-prostaglandin D2-DP1 receptor paracrine axis," Nature Immunology, vol. 14, no. 6, pp. 554-563, 2013.

[28] S. Bodhankar, A. A. Vandenbark, and H. Offner, "Oestrogen treatment of experimental autoimmune encephalomyelitis requires $17 \beta$,-oestradiol-receptor-positive $\mathrm{B}$ cells that upregulate PD-1 on CD4+ Foxp3+ regulatory T cells," Immunology, vol. 137, no. 4, pp. 282-293, 2012.

[29] V. Morales-Tirado, D. G. Wichlan, T. E. Leimig, S. E. A. Street, K. A. Kasow, and J. M. Riberdy, " $1 \alpha, 25$-dihydroxyvitamin D3 (vitamin D3) catalyzes suppressive activity on human natural regulatory $\mathrm{T}$ cells, uniquely modulates cell cycle progression, and augments FOXP3," Clinical Immunology, vol. 138, no. 2, pp. 212-221, 2011.

[30] M. Rouse, M. Nagarkatti, and P. S. Nagarkatti, "The role of IL2 in the activation and expansion of regulatory T-cells and the development of experimental autoimmune encephalomyelitis," Immunobiology, vol. 218, no. 4, pp. 674-682, 2013.

[31] S. Dimeloe, A. Nanzer, K. Ryanna, and C. Hawrylowicz, "Regulatory $\mathrm{T}$ cells, inflammation and the allergic response-The role of glucocorticoids and Vitamin D," Journal of Steroid Biochemistry and Molecular Biology, vol. 120, no. 2-3, pp. 86-95, 2010.

[32] S. Dhib-Jalbut and S. Marks, "Interferon- $\beta$ mechanisms of action in multiple sclerosis," Neurology, vol. 74, pp. S17-S24, 2010.

[33] M. K. Racke and A. E. Lovett-Racke, "Glatiramer acetate treatment of multiple sclerosis: an immunological perspective," Journal of Immunology, vol. 186, no. 4, pp. 1887-1890, 2011.

[34] V. Barnaba and V. Schinzari, "Induction, control, and plasticity of Treg cells: the immune regulatory network revised?" European Journal of Immunology, vol. 43, no. 2, pp. 318-322, 2013.

[35] A. Samanta, B. Li, X. Song et al., "TGF- $\beta$ and IL-6 signals modulate chromatin binding and promoter occupancy by acetylated FOXP3," Proceedings of the National Academy of Sciences of the United States of America, vol. 105, no. 37, pp. 14023-14027, 2008.

[36] A. Kimura and T. Kishimoto, "IL-6: regulator of Treg/Th17 balance," European Journal of Immunology, vol. 40, no. 7, pp. 1830-1835, 2010.

[37] E. Bettelli, Y. Carrier, W. Gao et al., "Reciprocal developmental pathways for the generation of pathogenic effector TH17 and regulatory T cells," Nature, vol. 441, no. 7090, pp. 235-238, 2006.

[38] W. A. Goodman, A. D. Levine, J. V. Massari et al., "IL-6 signaling in psoriasis prevents immune suppression by regulatory T cells," Journal of Immunology, vol. 183, no. 5, pp. 3170-3176, 2009.

[39] W. A. Goodman, A. B. Young, T. S. McCormick, K. D. Cooper, and A. D. Levine, "Stat3 phosphorylation mediates resistance of primary human T cells to regulatory T cell suppression," Journal of Immunology, vol. 186, no. 6, pp. 3336-3345, 2011.

[40] M. Murai, O. Turovskaya, G. Kim et al., "Interleukin 10 acts on regulatory $\mathrm{T}$ cells to maintain expression of the transcription factor Foxp3 and suppressive function in mice with colitis," Nature Immunology, vol. 10, no. 11, pp. 1178-1184, 2009.

[41] J. Klose, N. O. Schmidt, A. Melms et al., "Suppression of experimental autoimmune encephalomyelitis by interleukin-10 transduced neural stem/progenitor cells," Journal of Neuroinflammation, vol. 10, no. 1, p. 117, 2013. 


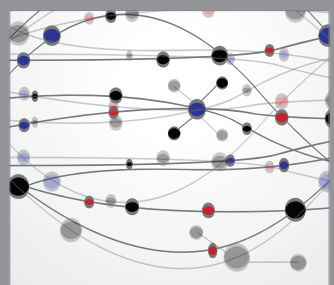

The Scientific World Journal
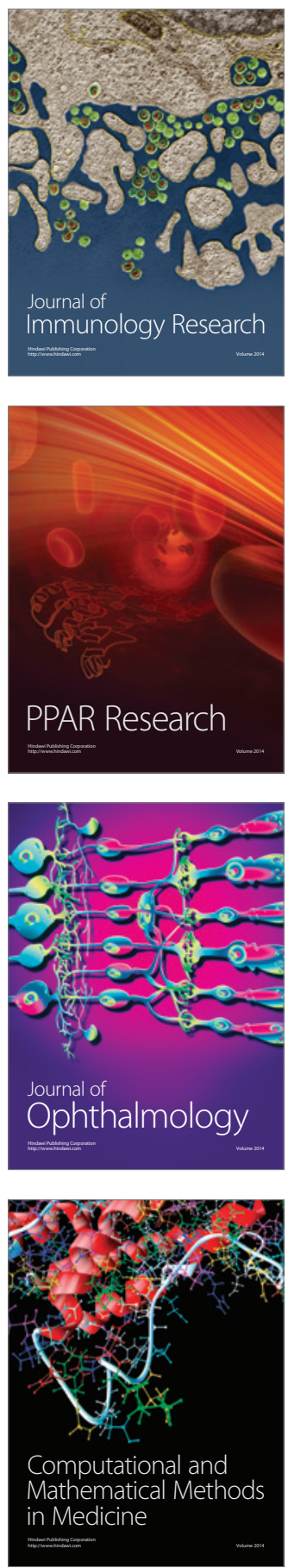

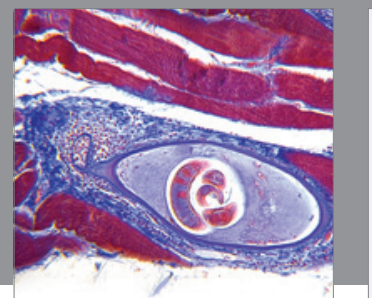

Gastroenterology

Research and Practice
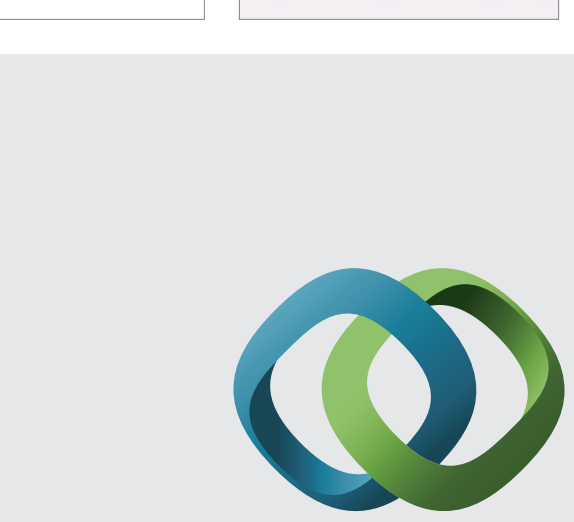

\section{Hindawi}

Submit your manuscripts at

http://www.hindawi.com
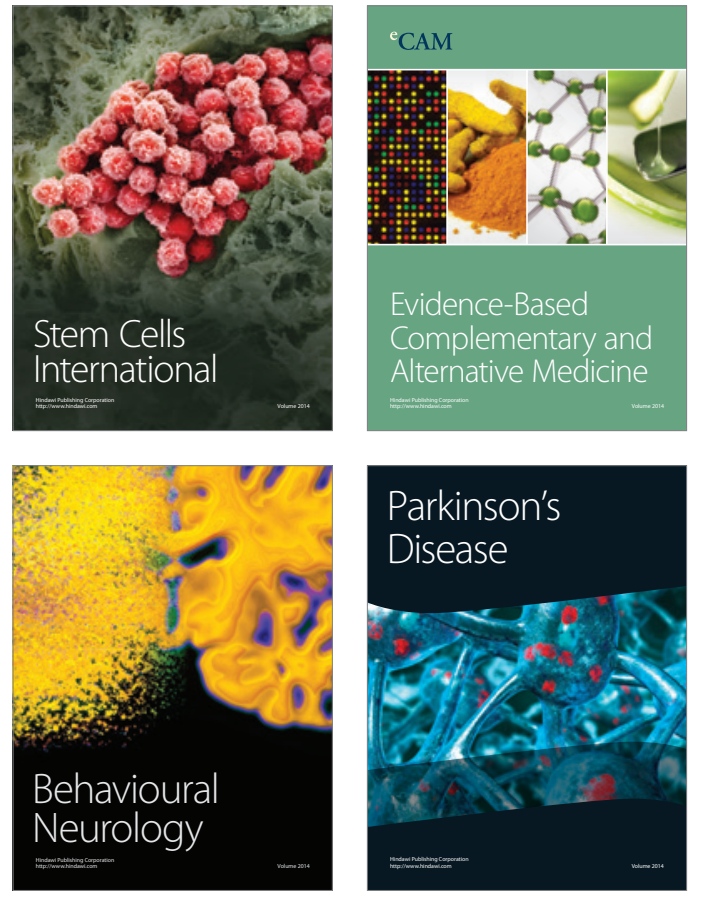
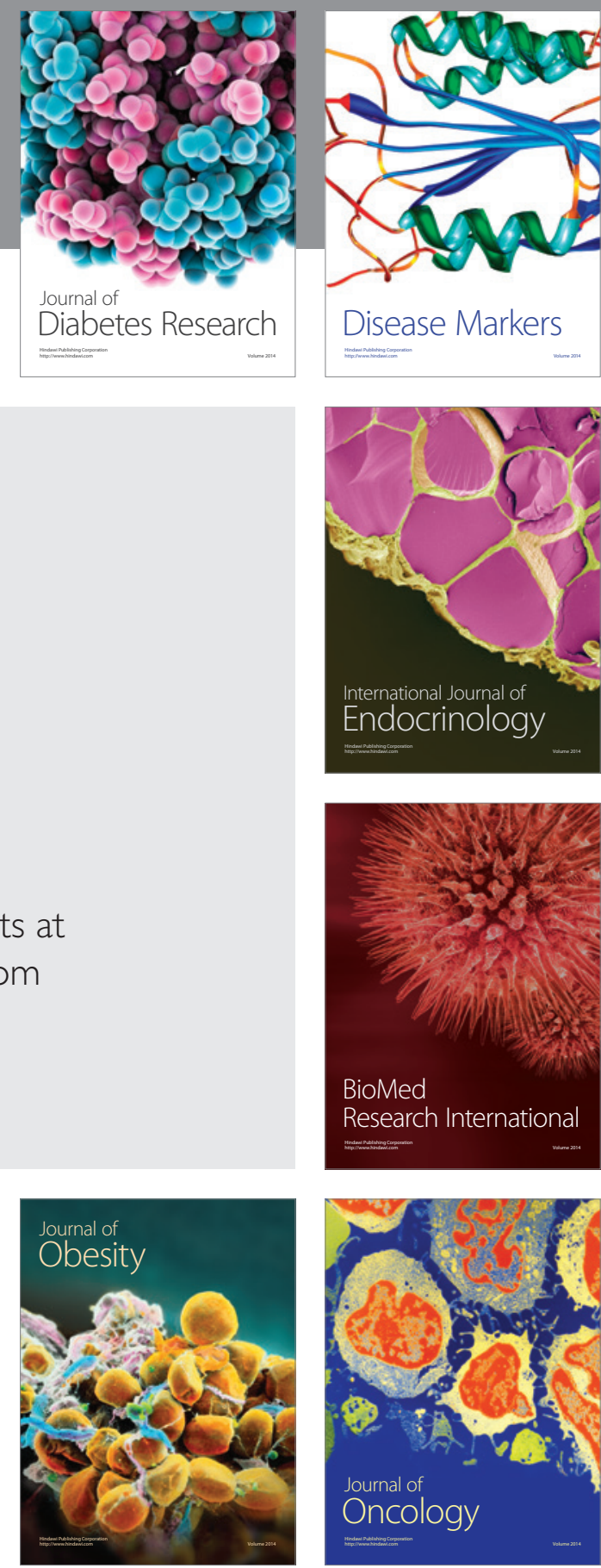

Disease Markers
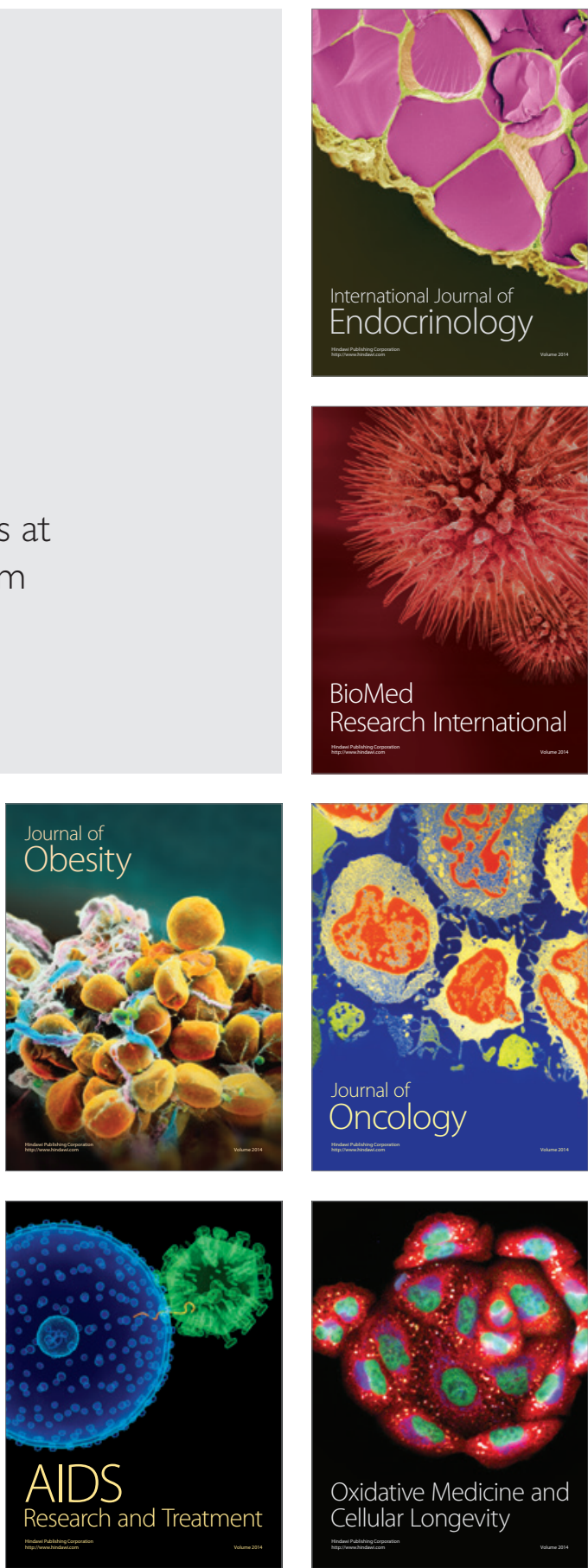